\title{
Erratum to: Training guidelines for endovascular stroke intervention: an international multi-society consensus document
}

\author{
S. D. Lavine ${ }^{1} \cdot$ K. Cockroft $^{1} \cdot$ B. Hoh ${ }^{1} \cdot$ N. Bambakidis ${ }^{1}$ - A. A. Khalessi ${ }^{1} \cdot$ H. Woo ${ }^{1} \cdot$ H. Riina ${ }^{1}$

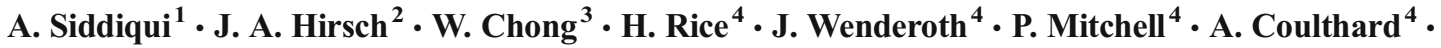

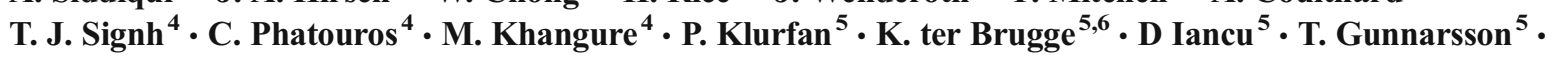

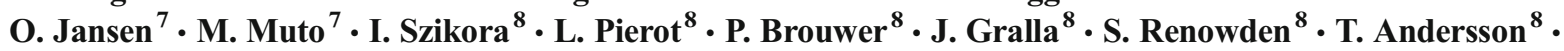

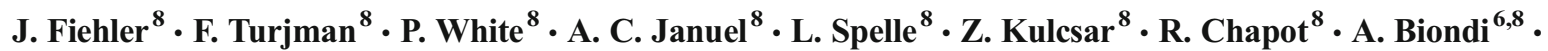

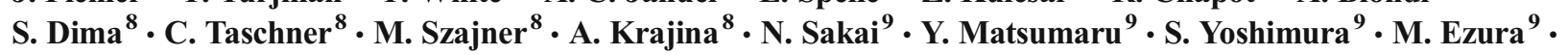 \\ T. Fujinaka ${ }^{9}$ K. Kihara ${ }^{9}$ - A. Ishii ${ }^{9}$ - T. Higashi ${ }^{9}$ - M. Hirohata ${ }^{9}$ • A. Hyodo ${ }^{9}$ - Y. Ito $^{9}$ • M. Kawanishi ${ }^{9}$ ' \\ H. Kiyosue ${ }^{9}$ - E. Kobayashi ${ }^{9}$ - S. Kobayashi ${ }^{9}$. N. Kuwayama ${ }^{9}$ - Y. Matsumoto ${ }^{9}$ - S. Miyachi ${ }^{9}$ Y. Murayama ${ }^{\text { }}$. \\ I. Nagata $^{9}$ - I. Nakahara ${ }^{9}$ - S. Nemoto ${ }^{9}$ - Y. Niimi ${ }^{9}$ - H. Oishi ${ }^{9}$ - J. Satomi ${ }^{9}$. T. Satow ${ }^{9}$. K. Sugiu ${ }^{9}$ - M. Tanaka ${ }^{6,9}$. \\ T. Terada ${ }^{9}$ - H. Yamagami ${ }^{9}$ - O. Diaz ${ }^{10}$. P. Lylyk $^{10}$ • M. V. Jayaraman ${ }^{11}$ - A. Patsalides ${ }^{11}$ - C. D. Gandhi ${ }^{11}$ \\ S. K. Lee ${ }^{11}$ T. Abruzzo ${ }^{11}$ - B. Albani ${ }^{11}$ - S. A. Ansari ${ }^{11}$ - A. S. Arthur ${ }^{11}$ - B. W. Baxter ${ }^{11}$ - K. R. Bulsara ${ }^{11}$. \\ M. Chen ${ }^{11}$ - J. E. Delgado Almandoz ${ }^{11}$ • J. F. Fraser ${ }^{11}$ • D. V. Heck ${ }^{11}$ • S.W. Hetts ${ }^{11}$ - M. S. Hussain ${ }^{11}$. \\ R. P. Klucznik ${ }^{11}$ - T. M. Leslie-Mawzi ${ }^{11}$ • W. J. Mack ${ }^{11}$ • R. A. McTaggart ${ }^{11}$ • P. M. Meyers ${ }^{11}$ - J. Mocco ${ }^{11}$. \\ C. J. Prestigiacomo ${ }^{11}$ - G. L. Pride ${ }^{11}$ • P. A. Rasmussen ${ }^{11}$ • R. M. Starke ${ }^{11}$ • P. J. Sunenshine ${ }^{11}$ • R. W. Tarr ${ }^{11}$ • \\ D. F. Frei ${ }^{11} \cdot$ M. Ribo ${ }^{12} \cdot$ R. G. Nogueira ${ }^{12}$ - O. O. Zaidat ${ }^{12} \cdot$ T. Jovin ${ }^{12} \cdot$ I. Linfante $^{12} \cdot$ D. Yavagal $^{12}$.

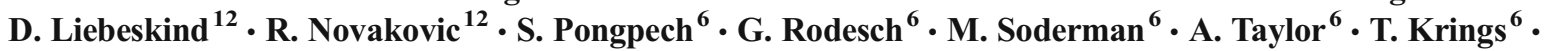

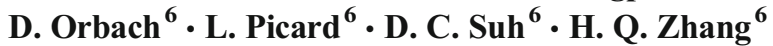

Published online: 20 June 2017

(C) Springer-Verlag GmbH Germany 2017

Erratum to: Neuroradiology (2016) 58:537-541

DOI 10.1007/s00234-016-1667-0

We note that there is an error in one of the authors' name. Author C Phatorous should have been C Phatouros.

The online version of the original article can be found at http://dx.doi.org/ $10.1007 / \mathrm{s} 00234-016-1667-0$

M. V. Jayaraman

mahesh.jayaraman@gmail.com

American Association of Neurological Surgeons/ Congress of Neurological Surgeons (AANS/CNS), Rolling Meadows, IL, USA

2 American Society of Neuroradiology (ASNR), Oak Brook, IL, USA

3 Asian Australasian Federation of Interventional and Therapeutic Neuroradiology (AAFITN), Bali, Indonesia

4 Australian and New Zealand Society of Neuroradiology - Conjoint Committee for Recognition of Training in Interventional Neuroradiology (CCINR) representing the RANZCR (ANZSNR), ANZAN and NSA, Sydney, Australia

5 Canadian Interventional Neuro Group (CING), Edmonton, AB, Canada
6 World Federation of Interventional and Therapeutic Neuroradiology (WFITN), Gold Coast, Australia

European Society of Neuroradiology (ESNR), Milan, Italy

8 European Society of Minimally Invasive Neurologic Therapy (ESMINT), Nice, France

9 Japanese Society for Neuroendovascular therapy (JSNET), Okayama, Japan

10 Sociedad Ibero Latino Americana de Neuroradiologica (SILAN), Cartagena, Colombia

11 Society of NeuroInterventional Surgery (SNIS), Washington, DC, USA

12 Society of Vascular and Interventional Neurology (SVIN), Brooklyn, NY, USA 